\title{
ERCP AND INTRAOPERATIVE LAPAROSCOPIC CHOLANGIOGRAPHY IN THE MANAGEMENT OF BILE DUCTS STONES - IS THERE AN ALTERNATIVE IN THE FACE OF MRCP
}

\author{
Ignatov V., K. Ivanov, N. Kolev, A. Tonev, M. Novakova ${ }^{1}$, R. Georgiev ${ }^{1}$ \\ Department of General and Operative Surgery, ${ }^{1}$ MRI-Division \\ University Hospital "St. Marina”
}

Reviewed by: Assoc. Prof. R. Madjov

\begin{abstract}
Background: It is controversial whether selective endoscopic sphincterotomy or routine laparoscopic bile duct exploration is the optimal treatment for choledocholithiasis. Magnetic resonance cholangio-pancreatography (MRCP) is a safe and accurate imaging modality; this study evaluated its use in a clinical algorithm for the management of suspected choledocholithiasis. Patients and methods: Consecutive patients presenting with suspected common bile duct (CBD) stones were managed according to an algorithm involving the selective use of MRCP to identify patients who required endoscopic sphincterotomy and bile duct clearance. Following radiological demonstration of a clear CBD, all patients were considered for cholecystectomy. Results: From 157 consecutive patients, 68 proceeded straight to endoscopic sphincterotomy, which was therapeutic in 59. Of 3 who underwent MRCP, choledocholithiasis or tumor obstruction was demonstrated; subsequent endoscopic sphincterotomy was therapeutic. Seventy-four patients subsequently underwent cholecystectomy, with a conversion rate of $9 \%$ and a median postoperative stay of 2 day. There were no instances of post-sphincterotomy pancreatitis or haemorrhage requiring transfusion. Conclusion: An algorithm involving selective MRCP with endoscopic sphincterotomy is a safe, effective means of managing suspected choledocholithiasis, particularly where the expertise, equipment or theatre time for laparoscopic bile duct exploration is not routinely available MRCP may become a new additional alternative to ERCP and intraoperative cholangiography.
\end{abstract}

Key words: Choledocholithiasis, MRCP, ERCP

\section{INTRODUCTION}

Common bile duct (CBD) stones are present in some 12\% of patients with symptomatic gallstone disease. In the majority of cases, choledocholithiasis is predictable on the basis of clinical, biochemical and radiological examination; however, a small proportion of patients have unsuspected CBD stones. There is considerable debate over whether intraoperative imaging of the CBD should be performed in all patients or only in those with suspected CBD stones. There is also controversy over whether CBD stones are optimally treated by preoperative endoscopic sphincterotomy or by intraoperative laparoscopic exploration of the CBD. Magnetic resonance cholangio-pancreatography (MRCP) is a safe, non-invasive means of imaging the biliary tree, whose accuracy for detecting CBD stones approaches $100 \%$ (1). Although not yet widely available, MRCP has been assessed prospectively as a means of selecting patients with $\mathrm{CBD}$ stones for preoperative endoscopic

Address for correspondence:

Kr.Ivanov, Varna, $1 \mathrm{Hr}$. Smirnenski str,

University Hospital "St. Marina"

e-mail: teraton@abv.bg, kivanov@gisurgery.bg sphincterotomy $(2,3)$; it is suggested that this approach might both avoid the need for intraoperative CBD imaging and reduce the unnecessary endoscopic sphincterotomy rate. Frimley University Hospital "St. Marina" a population of 800 000; gallstone disease is managed by two specialist upper GI surgeons who perform endoscopic retrograde cholangio-pancreatography (ERCP). All patients with suspected CBD stones are managed according to a clinical algorithm involving the selective use of MRCP to determine whether patients require preoperative clearance of the $\mathrm{CBD}$ by endoscopic sphincterotomy. Once the CBD is clear of stones, cholecystectomy is performed according to medical fitness and patient choice. This retrospective study reports the application of selective MRCP, endoscopic sphincterotomy and cholecystectomy in all patients admitted with suspected CBD stones during the year 2004.

\section{MATERIAL}

All patients presenting to University Hospital "St. Marina" with gallstone-related disease in the year 2001-2008 were managed according to a clinical algorithm accepted in the clinic. Previous and current clinical, biochemical and radio- 
logical indices were used to assess the need for preoperative evaluation of the CBD. Where there was a low suspicion of previous or current $\mathrm{CBD}$ stones, patients proceeded directly to cholecystectomy. Those with a high suspicion of CBD stones (CBD stones on ultrasound, cholangitis, pancreatitis, current elevated liver function tests LFTs) proceeded straight to ERCP and sphincterotomy. Equivocal cases underwent MRI cholangio-pancreatography, and the results of this were used to select patients for subsequent ERCP and sphincterotomy. Once the CBD was confirmed clear of stones, all patients were considered for cholecystectomy, and an informed decision was taken according to age, coexisting medical conditions and patient preference. Hospital discharge data, a computerized radiology database, endoscopy procedure records, the pathology database and operating logbooks were inspected to find all patients who underwent ERCP, MRCP or cholecystectomy during the year 2001-2008. ERCP was carried out by one of two specialist upper GI surgeons. Sedation (midazolam), analgesia (fentanyl) and anti-spasmodics (hyoscine butylbromide) were routinely given, and monitoring was in accordance with BSG guidelines. A side-viewing scope was used to visualize the ampulla, and retrograde cholangiography was performed, using a precut sphincterotomy if required. Gallstones were removed using sphincterotomy, balloon trawling and Dormia basket. In patients at risk of retained or recurrent CBD stones, a plastic stent was deployed to facilitate biliary drainage; this was removed or replaced as required after 6 weeks. Laparoscopic cholecystectomy was normally performed by one of two specialist upper GI surgeons or their supervised trainees. A Veress needle was used to establish a $12 \mathrm{mmHg}$ capnoperitoneum, and a standard four-port technique was used to dissect out Calot's triangle using hook diathermy and a grasper as required. After clipping and dividing the cystic duct and artery, the gallbladder was excised from the liver bed using hook diathermy and removed via the umbilical port, in a retrieval bag if necessary. Intra-abdominal drains were routinely placed, and patients were normally discharged home when eating, mobilizing and sufficiently analgesed. Quantitative data are expressed as mean, median or percentages. Ethics committee approval was not required for this study, nor was any statistical manipulation necessary.

\section{RESULTS}

A total of 132 patients with clinical, biochemical or radiological suspicion of choledocholithiasis were admitted to in the year 2008. Median age was 65 years (range 20-99) and $82(69 \%)$ were female. More than 223 further patients presented with cholelithiasis during the suspicion of CBD stones. These were considered at low risk of CBD stones, and are not considered further. Seventy-tree patients were considered at high risk of CBD stones, and proceeded straight to ERCP and sphincterotomy; cannulation of the CBD was achieved in 65 of these (92\%), and gallstones were demonstrated in $61(88 \%)$. The CBD was conclusively cleared in $52(71 \%)$ patients with CBD stones, while a plastic stent was placed in the remaining 9 patients in whom recurrent or retained stones could not be excluded. The remaining 8 patient, following failed ERCP, underwent laparoscopic CBD exploration, with successful removal of stones from the CBD.

In our hospital we established an MRI unit in MAY, 2008. Since then we evaluate 3 patients by MRCP in cases of unsuccessful ERCP. We menage to diagnose them - in those cases MRCP was a good alternative of the ERCP. Selective use of MRCP could be considered to have limited the number of patients undergoing ERCP from 65 to 61, and thus reduced the negative ERCP rate from $48 \%$ to $16 \%$. There were no incidences of post-ERCP pancreatitis or haemorrhage requiring transfusion; one patient (aged 78) with overwhelming sepsis due to ascending cholangitis died 2 days following successful ERCP and sphincterotomy.

\section{DISCUSSION}

This paper reports on the clinical application of a simple algorithm for the management of suspected CBD stones, and demonstrates that selective MRCP, with subsequent ERCP and laparoscopic cholecystectomy where appropriate, is a safe, successful and effective means of treating this common clinical problem. The incidence of CBD stones among patients undergoing cholecystectomy is reported as $12 \%$. In some cases, their presence is suspected on the basis of clinical history and examination; in others, abnormal liver function tests or a dilated CBD at ultrasound are the only clue, while a small percentage of patients have 'silent' or unsuspected CBD stones. Both before and since the advent of minimally invasive surgery, there has been controversy over whether the biliary tree should routinely be imaged during cholecystectomy. Some surgeons perform IOC in all patients, while others do so only in patients where there is clinical, biochemical or radiological evidence of CBD stones. Furthermore, there is considerable confusion over the optimum means of treating CBD stones; in some centres the preferred method is ERCP with sphincterotomy, while other centres undertake laparoscopic exploration of the CBD during cholecystectomy. Finally, there is uncertainty over whether the gallbladder should be removed following endoscopic clearance of the CBD, or should sphincterotomy be considered the definitive treatment. Firstly there is potential for unsuspected CBD stones in every patient presenting for cholecystectomy, and so routine imaging is the only way of establishing that the CBD is clear. However, a more selective approach is supported by evidence that only $1 \%$ of patients with normal liver function tests and normal calibre bile duct at ultrasound harbour stones in the CBD (4), and that over three-quarters of CBD stones pass spontaneously $(5,6)$. Cholangiography has a failure rate, and can give false negatives and false positives, leading to missed CBD stones and to unnecessary exploration of the $\mathrm{CBD}$, respectively (7). It also adds a certain 
amount of time to the operation, partly in surgical dissection and cannulation, and partly related to radiography (8). Secondly, it is claimed that cholangiography accurately delineates the biliary anatomy, thus reducing the risk of bile duct injury. This controversy is discussed in a recent review (9); although the largest population study suggests that routine IOC does reduce the risk of bile duct damage by about a third (10), such studies are open to bias, and there are no randomized controlled studies demonstrating a link. There is stronger evidence that routine IOC enables earlier detection of injury to the CBD, which may lead to improved outcome following appropriate early repair (11). However, reports from other centres where IOC is not routinely performed have shown that the most effective means of preventing CBD injury is meticulous dissection with correct interpretation of the anatomy (12). The clinical application of routine laparoscopic CBD exploration has recently been described (13), along with an exhaustive review of the literature comparing this approach with endoscopic sphincterotomy (14). The authors report 224 consecutive patients with CBD stones who were deemed fit for laparoscopic cholecystectomy; the overall CBD clearance rate was $96 \%$, with higher clearance rates following the addition of lithotripsy to the armamentarium. However, the rate of conversion to open operation was $13 \%$, with over half of these attributed to the CBD exploration, and the postoperative complication rate was $19 \%$, which might be considered high in a group of patients of mean age 56 years. Also, the mean postoperative hospital stay in 158 patients undergoing transductal exploration was 4.8 days, considerably longer than in most centres performing elective or urgent laparoscopic cholecystectomy. Routine IOC and laparoscopic CBD exploration add an unpredictable amount of time to operative duration (8), which is important in hospitals where theatre time is at a premium. All of these factors make $128 \mathrm{~S}$. Mercer et al. laparoscopic CBD exploration unattractive for daycase surgery. In addition to these immediate drawbacks of laparoscopic CBD exploration, there are as yet no follow-up data to conclusively demonstrate the absence of long-term sequelae. In particular, it is uncertain whether stenosis of the CBD following dissection and suturing of a longitudinal choledochotomy might lead to further stone formation or tumorigenesis. Finally, it should be noted that 149 patients with CBD stones were excluded from this study as they were considered unfit for laparoscopic cholecystectomy; these patients underwent ERCP and duct clearance as definitive treatment.

ERCP with sphincterotomy is considered inferior by proponents of laparoscopic CBD exploration for several reasons. Firstly, ERCP is thought to have a lower rate of stone clearance than laparoscopic CBD exploration; however, analysis of the randomized trials comparing the two techniques showed a similar overall duct clearance rate, with some centres reporting a $100 \%$ duct clearance at ERCP (14). Each technique has a learning curve, and it is likely that despite the occasional difficulty or failure, experienced practitioners of either method will have a success rate approaching $100 \%$. Secondly, ERCP is associated with sev- eral complications, most notably pancreatitis, which is reported to occur in up to $7 \%$ of cases and has a definite mortality (15). Pancreatitis has occasionally been reported following laparoscopic CBD exploration, but only in cases where instruments have transgressed the sphincter of Oddi. However, the incidence of pancreatitis following ERCP and sphincterotomy is related to technical factors rather than to co-existent medical factors. Several large studies have shown that the risk is higher in young female patients, and increased by repeated cannulation of the pancreatic duct, performance of a pre-cut sphincterotomy, and failure to clear the CBD of stones $(16,17)$. Finally, ERCP and sphincterotomy is postulated by some to encourage permanent duodenal biliary reflux, leading to formation of further bile duct stones and the possible development of biliary malignancy. However, the few studies that report a significant incidence of recurrent gallstone formation after ERCP and sphincterotomy relate either to the formation of further pigment-type stones in patients whose original gallstones were pigment stones, or to patients with the gallbladder still in situ following endoscopic sphincterotomy (18). Also, although there is a reported incidence of malignancy following biliary enteric anastomoses (19), there is no clinical evidence that endoscopic sphincterotomy is associated with the increased development of biliary cancer; any such increase would of course be difficult to differentiate from the known carcinogenic effects of long-term cholelithiasis. Thus, although ERCP and sphincterotomy is considered by some to be risky, critical analysis of the literature spanning over 25 years suggests that when performed by experienced practitioners, it leads to a high rate of CBD clearance with minimal short- and long-term complications. In the series described here, comprising over 100 ERCPs, no significant complications occurred, and the only death was attributed to multi-organ failure caused by ascending cholangitis. MRCP is a non-invasive imaging method that is highly sensitive and specific for the detection of CBD stones. It is quick, safe, well tolerated by most people, and has few contraindications and no known morbidity or mortality. Most recent studies comparing MRCP with IOC or ERCP findings have confirmed that MRCP has an accuracy of _95\%; in particular the negative predictive value approaches $100 \%(1,20,21)$. Several reports comparing MRCP findings with those at subsequent ERCP have concluded that reliance upon MRCP might have reduced the equirement for ERCP and sphincterotomy by up to $75 \%(1,2)$. For these reasons, MRCP has been incorporated into the pathway for the investigation of suspected CBD stones at our institution. Apart from occasional claustrophobic patients, it has proved highly acceptable, and has been pivotal in reducing the rate of 'negative' ERCPs to a minimum. No patient whose MRCP demonstrated a clear $\mathrm{CBD}$ returned with symptomatic CBD stones throughout the duration of this study, confirming the high negative predictive value for MRCP. There are conflicting opinions as to whether the gallbladder should be removed following successful CBD clearance. Further incidences of gallstonerelated pathology have been reported in only $5 \_16 \%$ of patients whose gall- 
bladder is left in situ following ERCP and sphincterotomy, leading some to conclude that cholecystectomy is not required $(22,23)$. This contrasts with the findings of a randomized controlled trial involving 120 patients who, following CBD clearance, were randomly allocated to a wait-and-see approach or to cholecystectomy (24). Over a 2 -year period, $47 \%$ of patients allocated to the wait-and-see group presented with further gallstone-related symptoms and $37 \%$ required cholecystectomy, with a conversion rate of $55 \%$. Our policy is to select patients for cholecystectomy according to age, co-morbidities and patient choice; those for whom sphincterotomy is considered definitive treatment are quoted a 1 in 7 risk for further gallstone-related symptoms. Where cholecystectomy is to be performed, our and others' data suggest that it should be done as soon as possible, to avoid the risk of patients presenting with further gallstone-related symptoms whilst awaiting elective cholecystectomy (25). In the hands of specialist surgeons, such a policy is not associated with an increased risk of conversion or other complications. The conversion rate of $10 \%$ reported in this series compares well with published reports, particularly considering that patients with CBD stones have an increased degree of fibrosis, and thus have a higher risk of conversion to open surgery. MRCP in the management of CBD stones 129 Conclusion We have devised and implemented an algorithm that uses liver function tests and transabdominal ultrasound to divide patients with suspected CBD stones into three groups. Those with normal LFTs and CBD diameter require no further imaging; those with a high suspicion of current bile duct stones progress directly to ERCP; the remainder, where the CBD status is uncertain, undergo further investigation using MRCP. Patients in whom MRCP demonstrates CBD stones undergo ERCP with sphincterotomy, while those with a clear CBD require no further investigation. All patients are then considered for laparoscopic cholecystectomy, with the decision to operate based upon age, medical fitness and patient choice. The results of this policy, as described in this paper, demonstrate that it is a robust, safe, effective means of investigating and treating CBD stones, and we recommend it for use in other hospitals.

An algorithm involving selective MRCP with endoscopic sphincterotomy is a safe, effective means of managing suspected choledocholithiasis, particularly where the expertise, equipment or theatre time for laparoscopic bile duct exploration is not routinely available MRCP may become a new additional alternative to ERCP and intraoperative cholangiography.

\section{REFERENCES}

1. Topal B, Van de Moortel M, Fieuws S, Vanbeckevoort D, Van Steenbergen W, Aerts $\mathrm{R}$, et al. The value of magnetic resonance cholangiopancreatography in predicting common bile duct stones in patients with gallstone disease. $\mathrm{Br} J$ Surg 2003;/90:/42_7.
2. Dwerryhouse SJ, Brown E, Vipond MN. Prospective evaluation of magnetic resonance cholangiography to detect common bile duct stones before laparoscopic cholecystectomy. Br J Surg 1998;/85:/1364_6.

3. Ke ZW, Zheng CZ, Li JH, Yin K, Hua JD. Prospective evaluation of magnetic resonance cholangiography in patients with suspected common bile duct stones before laparoscopic cholecystectomy. Hepatobiliary Pancreat Dis Int 2003;/2: 576_80.

4. Welbourn CR, Haworth JM, Leaper DJ, Thompson MH. Prospective evaluation of ultrasonography and liver function tests for preoperative assessment of the bile duct. Br J Surg 1995;/82:/1371_3.

5. Tranter SE, Thompson MH. Spontaneous passage of bile duct stones: frequency of occurrence and relation to clinical presentation. Ann R Coll Surg Engl 2003;/85:/174_7.

6. Collins C, Maguire D, Ireland A, Fitzgerald E, O'Sullivan GC. A prospective study of common bile duct calculi in patients undergoing laparoscopic cholecystectomy: natural history of choledocholithiasis revisited. Ann Surg 2004;/239: 28 33.

7. Hammarstrom LE, Holmin T, Stridbeck H, Ihse I. Routine preoperative infusion cholangiography versus intraoperative cholangiography at elective cholecystectomy: a prospective study in 995 patients. J Am Coll Surg 1996;/182:/408_16.

8. El Shallaly G, Seow C, Sharp C, Mughrabi A, Nassar AH. Intraoperative cholangiography time in laparoscopic cholecystectomy: timing the radiographer. Surg Endosc 2005;/19: 1370_2.

9. Connor S, Garden OJ. Bile duct injury in the era of laparoscopic cholecystectomy. Br J Surg 2006;/93:/158 68 .

10. Flum DR, Dëllinger EP, Cheadle A, Chan L, Koepsell T. Intraoperative cholangiography and risk of common bile duct injury during cholecystectomy. JAMA 2003;/289:/1639_44.

11. Archer SB, Brown DW, Smith CD, Branum GD, Hunter JG. Bile duct injury during laparoscopic cholecystectomy: results of a national survey. Ann Surg 2001;/234:/549_58.

12. Wright KD, Wellwood JM. Bile duct injury during laparoscopic cholecystectomy without operative cholangiography. Br J Surg 1998;/85:/191_4.

13. Thompson MH, Tranter SE. All-comers policy for laparoscopic exploration of the common bile duct. Br J Surg 2002;/89:/1608_12.

14. Tranter SE, Thompson MH. Comparison of endoscopic sphincterotomy and laparoscopic exploration of the common bile duct. Br J Surg 2002;/89:/1495_504.

15. Bergman JJ, $\bar{R}$ auws EA, Fockens P, van Berkel AM, Bassuyt PM, Tijssen JG, et al. Randomised trial of endoscopic balloon dilation versus endoscopic sphincterotomy for removal of bile duct stones. Lancet 1997;/349:/1124_9.

16. Masci E, Toti G, Mariani A, Cürioni S, Lamazzi A, Dinelli M, et al. Complications of diagnostic and therapeutic ERCP: a prospective 
multicenter study. Am J Gastroenterol 2001;/96: 417 23.

17. Loperfido S, Angelini G, Benedetti G, Chilovi F, Costan F, De Berardinis F, et al. Major early complications from diagnostic and therapeutic ERCP: a prospective multicenter study. Gastrointest Endosc 1998;/48:/1_10.

18. Geenen DJ, Geenen JE, Jafri FM, Hogan WJ, Catalano MF, Johnson GK, et al. The role of surveillance endoscopic retrograde cholangiopancreatography in preventing episodic cholangitis in patients with recurrent common bile duct stones. Endoscopy 1998;/30:/18 20.

19. Strong RW. Late bile duct cancer complicating biliary-enteric anastomosis for benign disease. Am J Surg 1999;/177:/472_4.

20. Dalton SJ, Balupuri S, Guest J. Routine magnetic resonance cholangiopancreatography and intra-operative cholangiogram in the evaluation of common bile duct stones. Ann R Coll Surg Engl 2005;/87:/469_70.

21. Demartines $\bar{N}$, Elsner L, Schnabel K, Fried R, Zuber M, Harder F. Evaluation of magnetic resonance cholangiography in the management of bile duct stones. Arch Surg 2000;/135:/148_52.

22. Kwon SK, Lee BS, Kim NJ, Lee HY, Chae $\mathrm{HB}$, Youn SJ, et al. Is cholecystectomy necessary after ERCP for bile duct stones in patients with gallbladder in situ? Korean J Intern Med 2001;/16:/254 9.

23. Schreurs WH, Vles WJ, Stuifbergen WH, Oostvogel HJ. Endoscopic management of common bile duct stones leaving the gallbladder in situ. A cohort study with long-term followup. Dig Surg 2004;/21:/60_4.

24. Boerma D, Rauws EA, Keulemans YC, Janssen IM, Bolwerk CJ, Timmer R, et al. Wait-and-see policy or laparoscopic cholecystectomy after endoscopic sphincterotomy for bileduct stones: a randomised trial. Lancet 2002;/360:/761_5.

25. Mercer SJ, Knight JS, Toh SK, Walters AM, Sadek SA, Somers SS. Implementation of a specialist-led service for the management of acute gallstone disease. Br J Surg 2004;/91:/504_8. 